\title{
Verwaltung und grundbedürfnisorientierte Entwicklung: Das Beispiel Sambia
}

\author{
Von Reinhard Bodemeyer
}

\section{Verwaltungsstruktur und das Grundbedürfnis nach Partizipation}

Die entwicklungspolitischen Grundlinien der Bundesregierung für die dritte Entwicklungsdekade der Vereinten Nationen (1980-1990) räumen der Bekämpfung der absoluten Armut entwicklungspolitische Priorität ein und nennen dazu die verbesserte Grundbedürfnisbefriedigung als geeignetes Mittel. ${ }^{1}$

Die Diskussion darüber, was Grundbedürfnis sei, geht über die Berücksichtigung der materiellen Bedürfnisse zur physischen Existenzsicherung hinaus in den Bereich der immateriellen Bedürfnisse hinein, die ihrerseits in individuellen und kollektiven Kategorien der Sozialpsychologie faßbar sind. ${ }^{2}$ Schwefel/Satzinger weisen darauf hin, daß beide Arten der Grundbedürfnisse zueinander komplementär sind und darüber hinaus die Befriedigung des einen ohne die gleichzeitige Berücksichtigung des anderen keine Grundbedürfnisbefriedigung gewährleistet. ${ }^{3}$

Mit einer Anerkennung des Grundbedürfniskonzeptes (GBK) mag eine gewisse Klarheit in die entwicklungspolitischen Zielvorstellungen gekommen sein, wenn auch eine Operationalisierung noch genug Schwierigkeiten aufwirft. Eine Bewertung von Entwicklungsvorhaben - seien sie nationaler, bilateraler oder multilateraler Natur - wird jedenfalls erleichtert, da sie sich an einer quasi-neutralen Meßlatte orientieren kann. Ein Charakteristikum des Konzeptes liegt darin, daß es scheinbar unpolitisch ist: die Befriedigung von Grundbedürfnissen hat - schon ihrer Diktion nach - einen so fundamental-humanistischen Anspruch, daß ihr Wert an sich - ähnlich wie die Achtung der Menschenrechte - von niemandem ernsthaft in Zweifel gezogen werden kann und wird. Damit wird das Konzept aber auch leicht zum Alibi und droht, zum Etikett zu verkommen, weil wohl jedes Projekt, Vorhaben oder Programm irgendjemandes Grundbedürfnis befriedigt.

Ist mit einer Entscheidung für das GBK eine entwicklungspolitische Programmformulierung erleichtert worden, bleiben dennoch erhebliche Implementationsschwierigkeiten zu lösen. Dabei geht es teilweise um die Aufgabe, Versorgungsleistungen auf die Befriedigung von Grundbedürfnissen abzustimmen bzw. sie quantitativ zu erhöhen. So

1 Bundesministerium für wirtschaftliche Zusammenarbeit, Die entwicklungspolitischen Grundlinien der Bundesregierung. Bonn 1980, S. 7 f.

2 Zur Definition der Grundbedürfnisse vgl. International Labour Organisation, Employment, Growth and Basic Needs. Genf 1976, sowie dies., Meeting Basic Needs: Strategies for Eradication of Poverty and Unemployment. Genf 1977.

3 D. Schwefel/W. Satzinger, Kriterien zur Beurteilung der Grundbedürfnisrelevanz von Projekten. München (mimeo.) 1979, S. 7. 
sehen Burki/Ul Haq die Hauptaufgabe des GBK darin, die staatlichen sozialen Dienste auf bis dahin nicht erreichte Bevölkerungsgruppen auszudehnen. ${ }^{4}$ Abgesehen von der Kostenintensität eines so interpretierten GBK liegt diesem Vorschlag die Annahme zugrunde, jedes beliebige Entwicklungsland besäße eine Verwaltung, die als amorpher Dienstleistungsbetrieb lediglich neu zu programmieren sei, um eine Grundbedürfnisorientierung zu institutionalisieren.

Schneider-Barthold weist darauf hin, welche Bereiche der wirtschafts- und entwicklungspolitischen Ebene tangiert werden bei der Entscheidung, die Grundbedürfnisorientierung als Leitschnur des nationalen Entwicklungspfades anzunehmen. ${ }^{5}$ Die Verwaltungsstruktur erhält dabei eine zentrale Bedeutung, wie am Fall des Grundbedürfnisses nach Partizipation deutlich zu machen sein wird. Partizipation hat eine aktive und passive Wortbedeutung. Als passive Partizipation kann die Teilhabe an Leistungen des Staates im weitesten Sinne gelten. Aktive Partizipation schließt dagegen das Mitentscheiden über die Verteilung der Leistungen selbst ein. Das Grundbedürfniskonzept legt von seinem normativen Verständnis her den Akzent auf aktive Partizipation.

Eine zentralistische Verwaltungsstruktur ist unter Umständen geeignet, passive Partizipation zu gewährleisten. Die gleichmäßige Verteilung von Leistungen ist in erster Linie ein Problem der betriebswirtschaftlichen, inneradministrativen Optimierung. ${ }^{6}$

Aktive Partizipationen dagegen erfordert dezentrale Verwaltuangsstrukturen, die Entscheidungen zulassen, in denen die administrative Rationalität nicht der ausschließliche oder überwiegende Faktor ist.

Ein Bekenntnis zur grundbedürfnisorientierten Entwicklung erfordert bei gegebener überzentralisierter administrativer Struktur in den meisten Entwicklungsländern ${ }^{7}$ also eine Verwaltungsreform mit dem Ziel der Dezentralisierung.

Schon oben wurde das GBK als nur scheinbar unpolitisch charakterisiert. In dem Maße, wie es über Partizipation zu sozialer Emanzipation beiträgt, wird es in der Regel dazu führen, auch die gegebenen gesellschaftlichen Machtverhältnisse in Entwicklungsländern

4 S. J. Burki/M. Ul Haq, Meeting Basic Needs - an Overview. In: World Development, (1981)9, S. $167 \mathrm{ff}$.

5 W. Schneider-Barthold, Schwierigkeiten mit dem Grundbedürfniskonzept. In: Entwicklung und Zusammenarbeit, (1981)11, S. 12 ff.

6 Unter anderem wird der komparative Vorteil der Zentrale bei Verteilungsentscheidungen hervorgehoben. Vgl. P. Musgrave/R. A. Musgrave, Public Finance in Theory and Practice. Tokio 1976, S. 606.

7 Vgl. United Nations Centre for Regional Development, Senior Level Seminar on Implementing Decentralization Policies and Programmes, Report of UNCRD Seminar Held at Nagoya, Japan - 21 to 27 July 1981, 24 to 30 August 1982. Nagoya. Beide Reports enthalten Länderstudien zu den Verwaltungssystemen v. a. im pazifischen Raum. G. Benneh, Decentralisation and Rural Development in Ghana. In: African Administrative Studies, (1977)17, S. 45 ff. J. Craig/W. Reilly/W. Tordoff, Studies in Decentralisation: Papua New Guinea, Botswana, Sri Lanka. Manchester Papers on Development 3, Manchester 1981. D. A. Rondinelli, Government Decentralization in Comparative Perspective: Theory and Practice in Developing Countries. In: International Review of Administrative Sciences, 47 (1981)2, S. 133 ff. R. Kaufmann, Regionale Entwicklungskörperschaften als Instrumente der Einkommensübertragung aus der Stadt in den ländlichen Raum (Beispiel Bogotá/Kolumbien). In: R. Bodemeyer et al., Stadt-Land-Verflechtung und Einkommensverteilung in Entwicklungsländern. Saarbrücken 1981, S. 60 ff. Zur systematischen Begründung der Úberzentralisierung vgl. P. Mawhood, Stages of Development and the Model for Local Government Reform in Afrika. In: Mlinar/Teune (eds.), The Social Ecology of Change. London 1978, S. 210. 
durch die Bevölkerung in Frage stellen zu lassen. Das macht das GBK im Gegensatz zum Community Development Ansatz zur "politisierenden" Strategie. Wurde nun weiter argumentiert, die Umsetzung der Grundbedürfnisorientierung in nationale Entwicklungspolitik erfordere eine Verwaltungsreform mit dem Ziel der Dezentralisierung, und bezieht man ferner mit ein, daß jede Verwaltungsreform "power politics in action " ${ }^{8}$ ist, verliert das GBK seinen unpolitischen Charakter vollends.

Im folgenden soll gezeigt werden, in welchem Maße und auf welche Weise eine Grundbedürfnisorientierung die überkommenen Implementationsstrukturen der öffentlichen Verwaltung in Entwicklungsländern in Frage stellt und wie eine Verwaltungsreform einer grundbedürfnisorientierten nationalen Entwicklungspolitik Rechnung tragen kann. Der damit angesprochene Zusammenhang zwischen Implementationsstruktur und politischem Programminhalt wird anhand der Agrarpolitik diskutiert. Die abgeleiteten Hypothesen sollen am Fallbeispiel Sambia überprüft werden.

\section{Produktions- versus bedürfnisorientierte Agrarpolitik}

Es kann davon ausgegangen werden, daß die Landwirtschaft für die meisten Entwicklungsländer prioritäre Bedeutung besitzt. Der Begriff "prioritär" sei hier in einem umfassenden Sinne verstanden: er meint nicht nur die ökonomische Bedeutung des Sektors, gemessen an seinem Anteil am Bruttosozialprodukt, an der Beschäftigung und am Export, sondern erstreckt sich insgesamt auf die Bedeutung des Sektors im Entwicklungsprozeß. Die Einschätzung der Landwirtschaft ist in der neueren entwicklungspolitischen Diskussion einem Wandel unterworfen, der nicht losgelöst von der weltwirtschaftlichen Entwicklung zu begreifen ist. Ohne der Diskussion in ihrer ganzen Breite folgen zu wollen sei lediglich darauf verweisen, daß in erster Linie energiekostenbedingte Zahlungs- und Außenhandelsbilanzschwierigkeiten dazu beigetragen haben, der eigenen nationalen Landwirtschaft im Rahmen einer Politik der Selbstversorgung zur Reduktion von Nahrungsmittelimporten einen höheren Stellenwert einzuräumen. ${ }^{9}$ Darüber hinaus formuliert der Weltentwicklungsbericht positiv, daß die Landwirtschaft nicht nur zur Stärkung der gesamten Volkswirtschaft beitrage, sondern gleichermaßen helfe, die Armut im ländlichen Raum einzudämmen. ${ }^{10}$

Gewinnt damit die Landwirtschaft gegenüber den anderen volkswirtschaftlichen Sektoren eine subjektiv oder objektiv höhere Bedeutung, so ist die Agrarpolitik selbst zwei einander teilweise widersprechenden Zielvorstellungen ausgesetzt. Unter Inkaufnahme einiger Vereinfachungen kann die Agrarpolitik die landwirtschaftliche und/oder die

\footnotetext{
(i. E. Caiden, Administrative Reform. London 1970, S. 9.

9 Neben steigenden Energiekosten durch Olpreissteigerungen wirken niedrige Preise für industrielle Rohstoffe, die ihrerseits auf rezessionsbedingte Nachfrageausfälle der Industrieländer zurückgehen. Overseas Development Institute, Briefing Paper. (1982)2, S. 4.

10 Weltbank, Weltentwicklungsbericht 1982, Washington D. C. 1982, S. 45 ff.
} 
ländliche Entwicklung als prioritäres Ziel verfolgen, kann also produktions- und/oder bedürfnisorientiert sein. Es kann ferner davon ausgegangen werden, daß die Produktionserhöhung das klassische Ziel der Agrarpolitik ist, während die bedürfnisorientierte, ländliche Entwicklung einer historisch jüngeren Zielvorstellung entspricht. ${ }^{11}$

Die Einschätzung liegt nahe, daß produktions- und bedürfnisorientierte Agrarpolitik einander zumindest teilweise widersprechen. Diese Einschätzung ist insofern berechtigt, als produktionsorientierte Agrarpolitik sich in ihrer Förderung entweder selektiv an bereits modernisierten Produktionsbetrieben orientiert oder aber durch ihre Förderung selbst aktiv selektiert insofern, als sie nicht die ländliche Bevölkerung sondern bestimmte Agrarproduzenten als zu fördernde Zielgruppe implizit oder explizit anspricht und damit bestehende Unterschiede zementiert und ausweitet. ${ }^{12}$ Ländliche Entwicklung geht über die sozialproduktbezogenen Wachstumsziele hinaus und versucht ihrem Anspruch nach, "soziologisch-soziale" Ziele mitzuberücksichtigen. Sie orientiert sich also daran, die Lage der ländlichen Bevölkerung zu verbessern, wobei die Erhöhung der Agrarproduktion nur Teilziel der Entwicklungspolitik ist. ${ }^{13}$

Im folgenden wird die These vertreten, daß zur Unterscheidung der beiden Ziele der Agrarpolitik das Motiv des jeweiligen politischen Akteurs - in den meisten Fällen der Staat im Entwicklungsland - auszumachen ist. Auf dieser Ebene entspringt eine produktions-orientierte Agrarpolitik meist unmittelbar drängenden und kurzfristigen Zielen der städtischen Versorgung mit Nahrungsmitteln bzw. der Versorgung der heimischen Industrie mit Rohstoffen, während bedürfnisorientierte, ländliche Entwicklung eher dem Ziel der langfristigen Legitimationsbeschaffung oder -sicherung des jeweiligen Regimes dient. Insofern ist eine Orientierung der Agrarpolitik auf Bedürfnisbefriedigung oder Produktionssteigerung nicht Gegenstand einer willkürlichen Alternativenwahl sondern entspringt dem lang- oder kurzfristigen Kalkül der jeweiligen Staatsführung. Zudem können beide Ziele durchaus simultan verfolgt werden, wie das Fallbeispiel Sambia zeigt.

Ist nun aber unter (1.) bereits angedeutet worden, daß eine gegebene Verwaltungsstruktur nicht durchweg geeignet zur Implementation von Programmen jeden beliebigen politischen Inhalts ist, kann die Fragestellung dahingehend zugespitzt werden, welcher Typus von Verwaltungsstruktur einer Implementation grundbedürfnisorientierter Programme der Agrarpolitik förderlich ist.

11 J. v. Stockhausen, Zur Verknüpfung von Entwicklungsstrategie mit ihren Trägerstrukturen - diskutiert am Beispiel des Agrarkredits. In: Entwicklung und ländlicher Raum, (1982)6, S. 17.

12 A. Bartelink, Verfahrensweise bei der Arbeit mit Zielgruppen für die landwirtschaftliche Beratung in Entwicklungsländern - ein differenzierter Ansatz am Beispiel der Provinz Misiones, Argentinien. Giessen (mimeo.) 1982, S. 2.

13 S. Schönherr, Konzeptionen ländlicher Entwicklungspolitik. In: Zeitschrift für ausländische Landwirtschaft, (1979)1, S. 5. 


\section{Staatselite und Bürokratie in Entwicklungsländern}

Eine solche Frage kann ohne empirischen Hintergrund nicht diskutiert werden. Die Empirie zeigt aber, daß aus verschiedenen noch zu erörternden Gründen die Struktur der öffentlichen Verwaltung in Entwicklungsländern überwiegend zentralistisch ist. ${ }^{14}$

Dieses Phänomen erklärt sich nicht zuletzt aus der Entwicklungspolitik der ersten beiden Entwicklungsdekaden: eine der Modernisierung verpflichtete Entwicklungspolitik, die Entwicklung mit Industrialisierung gleichsetzt, bringt eine städtisch orientierte, hierarchisch-elitäre, vertikal durchorganisierte Verwaltung hervor, die zentrale, "moderne" Entscheidungen und Programme gegen traditionelle Ansichten und Einstellungen durchsetzt. Die subjektive Qualität der Verwaltung bestimmt sich dann nach dem Grad der Penetration des jeweiligen Landes mit ihren Institutionen. ${ }^{15}$ Die Bürokratie ist dabei kein eigeninteressenloses Implementationsinstrument.

In den meisten Entwicklungsländern enthält die Bürokratie zum Zeitpunkt der Unabhängigkeit eine relativ große und homogene Gruppe der autochthonen Bevölkerung, die im Vergleich zur Restbevölkerung relativ qualifiziert ausgebildet ist. ${ }^{16}$ Das führt in vielen Fällen bereits vor der Unabhängigkeit zumindest zu sozialer Stratifizierung wenn nicht zur Bildung eines ausgeprägten Elitebewußtseins unter den autochthonen Angehörigen der Bürokratie. Die relative Privilegierung dieser Elite trägt dazu bei, daß sie nur in Ausnahmefällen zu einer aktiven Unterstützung der Unabhängigkeitsbewegung bereit ist. Nichtsdestoweniger macht ihre Bildung und Ausbildung sie zum eigentlichen Verbündeten der Staatsführung nach der Unabhängigkeit. Diese Koalition hat ihren Preis in einer Privilegierung der Angehörigen der zentralen Bürokratie, die bis zur Besetzung politischer Ämter wie die Leitung von Ministerien mit Kabinettsrang durch Bürokraten reicht, da der administrative Sachverstand unter allen Umständen loyal erhalten werden muß.

Inneradministrativ erhält dadurch die Zentrale einen überdurchschnittlich hohen Stellenwert gegenüber den nachgeordneten Verwaltungen. Sie verfügt über vergleichsweise bessere formale Qualifikation und über wesentlich bessere materielle Absicherung. Die Dominanz der Zentrale hat darüber hinaus Konsequenzen für die Verwaltungsstruktur insofern, als Ressort-Ministerien das Patronage-System intern übernehmen und dadurch

14 Stellvertretend D. A. Rondinelli, Government Decentralisation in Comparative Perspective ..., a.a.O. S. 133. M. Bratton, The Local Politics of Rural Development. Hannover N. H./London 1980, S. 16. Vgl. auch Anm. 7.

15 Stellvertretend für die modernistische Interpretation von Staat und Bürokratie in der Dritten Welt: S. Eisenstadt, Political Development. In: A. Etzioni/E. Etzioni (eds.), Social Change. New York 1964, S. 310 ff. Zur Kritik der Interpretation: D. A. Rondinelli/K. Ruddle, Appropriate Institutions for Rural Development: Organizing Services and Technology in Developing Countries. In: Philippine Journal of Public Administration, II (ohne Jahr)I, S. 35 ff. M. Bratton, The Local Politics of Rural Development, a.a.O. S. 11. B. C. Chikulo, Popular Participation and Development: the Zambian Model. In: African Quarterly (New Dehli), 19(1979)2, S. 179.

16 I. Scott, Party and Administration under the One-Party State. In: W. Tordoff (ed.), Administration in Zambia. Manchester 1980, S. 141 
Ressortorientierung fördern, die ihrerseits die sektorale, vertikale Ausdifferenzierung der Bürokratie zur Folge hat. ${ }^{17}$

Ist damit noch die Herausbildung eines vertikal einheitlichen Professionalismus unwahrscheinlich, so ist dennoch die Verbreitung einer städtisch-modernistischen Interpretation von Entwicklung als gemeinsame Werthaltung innerhalb der Bürokratie zu erwarten. Eine so geprägte Verwaltung verhält sich gegenüber politischen Programmen mit Grundbedürfnisorientierung keineswegs indifferent. Sie wird mit der Aufgabe betraut, Programme zu implementieren, deren Hintergrund und Inhalt einen städtisch-modernistischen Entwicklungspfad, mithin also ihr eigenes Selbstverständnis und - nicht zuletzt - die eigene materielle Basis hinterfragt.

Die fiktiv-autonome politische Führung in Entwicklungsländern verfügt also keineswegs über eine Bürokratie, die als eigeninteressenloses Implementationsinstrument beliebig programmierbar ist. Die Umsetzung von Grundbedürfnisorientierung in nationale Programme macht im Gegenteil eine Verwaltungsreform erforderlich, die über eine rein binnenadministrative Reorganisation weit hinausreicht und meist erhebliche innenpolitische Konsequenzen hat.

\section{Verwaltungsdekonzentration als Strategie zur Verbesserung der Implementationsfä- higkeit}

Eine Strategie, die sich zur Anpassung der Implementationsstrukturen an veränderte Steuerungserfordernisse anbietet, ist die Dekonzentration der Verwaltung. Dazu lassen sich im wesentlichen drei Begründungszusammenhänge anführen.

(1) Auf der binnenadministrativen Ebene spricht vor allem eine Erhöhung der Flexibilität und eine verbesserte Informationsverarbeitung für Dekonzentration. Flexibilität ist dann erforderlich, wenn eine Bedürfnisorientierung der Programme problemgerechtes über normgerechtes Entscheidungsverhalten stellt. Ein solches Entscheidungsverhalten setzt Regelungsbefugnisse der nachgeordneten Institutionen voraus.

Informationsverarbeitung wird dadurch wichtig, daß neben dem vertikalen Informationsfluß von oben nach unten (Charakteristikum für vorwiegend distributives staatliches Handeln) die Informationsweitergabe von unten nach oben sowie horizontale Kommunikationsstrukturen an Bedeutung gewinnen. Letzteres hat besonderes Gewicht für ländliche Entwicklung, da die Aufgabe meist ressortübergreifend organisiert ist und Koordination an der Basis notwendig macht.

Obwohl Dekonzentration im allgemeinen den Bedarf an qualifiziertem Personal vergrößert (eine kritische Größe vor allem in afrikanischen Entwicklungsländern), ist dennoch eine erhebliche Steigerung der administrativen Effizienz zu erwarten. Die Kosten von

17 D. Dresang, The Zambia Civil Service - Entrepreneurialism and Development Administration. Nairobi 1975, S. 161 . 
Dekonzentrationsprogrammen sollten daher als produktivitätssteigernde Investitionen aufgefaßt werden.

(2) Für eine produktionsorientierte Agrarpolitik ergeben sich durch administrative Dekonzentration im wesentlichen die Vorteile, die unter (1) als Verbesserung des administrativen Outputs charakterisiert wurden. So ist eine bessere Abstimmung der einzelnen Faktoren innerhalb komplexer Förderungsprogramme zu erwarten, kürzere Zuständigkeitswege und dadurch verkürzte Bearbeitungszeiten, bessere Bedarfsermittlung sowie, wiederum, höhere Flexibilität, die sich aus einer Verfügbarkeit entwicklungsrelevanter Ressourcen vor Ort ergibt.

(3) In bezug auf ländliche Entwicklung, innerhalb derer die Erhöhung der Agrarproduktion nur Teilbereich ist, erlaubt eine Dekonzentration darüber hinaus die aktive Partizipation der ländlichen Bevölkerung. Dekonzentrierte Verwaltungsstrukturen sind deswegen erforderlich, da es ja nicht um die schlichte Verteilung von Ressourcen anhand zentral formulierter Kriterien geht, sondern im Gegenteil eine Bedürfnisorientierung eine Bedürfnisermittlung voraussetzt.

Im Gegensatz zur unter (1) und (2) abgeleiteten administrativen Dekonzentration erfordert aktive Partizipation zusätzlich eine politische Dezentralisierung, d. h. eine Verlagerung politischer Kontrolle, um der Verwaltung auf lokaler Ebene ein repräsentatives Kontrollorgan zur Seite stellen zu können. ${ }^{18}$

Aktive Partizipation ist einerseits Ziel in sich selbst im Rahmen des Grundbedürfniskataloges. Sie ist darüber hinaus Instrumentalziel für gesellschaftliche und soziale Entwicklung sowie zur Legitimationsbeschaffung des politischen Systems. Sie ist ferner ein Instrument der Egalisierung insofern, als sie ein Korrektiv gegenüber willkürlichen Verteilungsentscheidungen sein kann. Sie schafft eine Klientelverpflichtung der Verwaltung gegenüber der Bevölkerung und ist langfristig die effektivste Art, ökonomischen und sozialen Fortschritt zu erreichen. ${ }^{19}$

\section{Restriktionen der Dezentralisierung}

Die unter (4.) genannten Vorzüge einer dekonzentrierten Verwaltung sind im Prinzip weder neu noch umstritten. Dezentralisierung - und damit Dekonzentration - ist seit langer Zeit Grundtenor in Konzeptionspapieren der Vereinten Nationen zur Entwicklungsverwaltung und in den meisten wissenschaftlichen Empfehlungen zum gleichen Thema. ${ }^{20}$ Dennoch wird das Konzept nur sehr zögernd, in einigen Fällen überhaupt nicht

18 P. Mawhood, Negotiating from Weakness: the Search for a Model of Local Government in Countries of the Third World. In: Planning and Administration, (1974)1, S. $22 \mathrm{ff}$.

19 B. C. Chikulo, Popular Participation and Development ..., a.a.O., S. $173 \mathrm{f}$.

20 United Nations, Decentralisation for National and Local Development. New York 1962, S. 2 ff. D. A. Rondinelli/K. Ruddle, Urbanization and Rural Development - a Spatial Policy for Equitable Growth. New York 1978, S. 39, 115 und 119. United Nations Centre for Regional Development, Newsletter No. 11, Januar 
in die Praxis umgesetzt. Dazu wird hier die These vertreten, daß die genannten Empfehlungen von Restriktionen absehen, die sich aus den politisch-gesellschaftlichen Rahmenbedingungen ergeben, die für Entwicklungsländer charakteristisch sind.

Dezentralisierung führt wie jede Verwaltungsreform, zumal wenn sie die Verlagerung von politischer Kontrolle einschließt, zu erheblichen Machtverschiebungen nicht nur zwischen zentraler und lokaler Ebene, sondern auch zwischen Bürokratie und jeweiliger politischer Führung sowie zwischen Fraktionen und Interessengruppen innerhalb der Staatselite, wobei die Fraktionsgrenzen durchaus quer zu den Apparatsgrenzen liegen können.

Um hier zu konreten Aussagen zu gelangen, kann teilweise auf das oben dargestellte Verhältnis zwischen Bürokratie und Staatselite zurückgegriffen werden. In dem Maße, wie sich die Bürokratie als Bündnispartner für die Staatselite unentbehrlich macht, in dem Maße partizipiert sie auch an der zentralen Machtausübung. Dezentralisierung bedeutet nicht nur individuellen Statusverlust und materielle Einbuße der in die Provinz transferierten Beamten sondern ist geeignet, die zentrale Durchsetzungsfähigkeit der Bürokratie als Machtgruppe insgesamt zu gefährden. Daher kann ein erheblicher Widerstand gegen Dezentralisierung innerhalb der Bürokratie selbst vermutet werden. Oben ist argumentiert worden, die vertikale und hierarchisch-zentralistische Verwaltungsstruktur entspräche einer städtisch-modernistischen, auf Industrialisierung ausgerichteten Entwicklung. Wird nun angenommen, daß eine Verwaltungsdezentralisierung der Implementation einer grundbedürfnisorientierten Politik dient, so kann gleichzeitig angenommen werden, daß die Verwaltung damit an Eignung für die Durchsetzung zentral formulierter städtisch-industrieller Programme verliert. In dem Maße also, wie eine grundbedürfnisorientierte, ländliche Entwicklung innerhalb der Staatselite selbst umstritten ist, in dem Maße werden sich auch Gegner und Befürworter einer Verwaltungsdezentralisierung unterscheiden lassen. ${ }^{21}$

Schließlich geht mit Dezentralisierung der Verwaltung meist der Verlust an zentraler Kontrolle einher, der die politische Stabilität des Systems möglicherweise gefährdet. Selbst wenn Dezentralisierung ökonomisch rational ist, wird sie häufig zulasten der politischen Kontrolle gehen und deshalb vermieden werden. ${ }^{22}$

Eher technischer Natur ist der Hinweis, daß Dezentralisierungsprogramnie selber ein hohes Maß an Eigenkomplexität aufweisen, die erhebliche Anforderungen an Koordination und organisatorische Effizienz stellt. ${ }^{23}$ Dazu ist administrativer Sachverstand unabdingbar, der seinerseits meist nur innerhalb der Bürokratie vorhanden ist. Para-

1981, Nagoya, S. 4. H. G. Braun, Concepts and Problems of Decentralized Industrialization in Developing Countries. In: Economics, (1981) 24, S. 71

21 P. Ollawa, Participatory Democracy in Zambia: the Political Economy of National Development. Elmscourt 1979, S. 350.

22 V. C. Uchendu, Comment on L. Gray Cowan: The Political and Administrative Setting for Rural Development. In: F. S. Arkhurst (ed.), A frica in the Seventies and Eighties - Issues in Development. New York 1970, S. 126.

23 J. Fesler, Area and Administration. University of Alabama 1971, S. 65. 
doxerweise stößt also ein Programm, das die Implementationskapazität der Verwaltung verbessern soll, selbst auf Implementationsprobleme, die zudem von einer Bürokratie gelöst werden sollen, die dem Programm selbst oft ablehnend gegenüber steht.

\section{Das sambische Dezentralisierungsprogramm}

Ziel des sambischen Dezentralisierungsprogrammes von 1981 ist eine beschleunigte ländliche Entwicklung. ${ }^{24}$ Es sind im wesentlichen vier Gründe, die ein verstärktes Interesse der Staatsführung am ländlichen Raum bedingen.

Eher moralisch-ethischer Natur ist die Verpflichtung, die sich aus der of fiziellen sambischen Philosophie des Humanismus ableitet, der der Entwicklung des Menschen Vorrang vor abstrakten Wachstumszielen einräumt, und insofern stark an das Grundbedürfniskonzept erinnert. ${ }^{25}$

Ein gewisser Druck geht sicher auch auf die Beratungstätigkeit der Internationalen Labour Organisation (ILO) in Sambia zurück, die dem Grad der Grundbedürfnisbefriedigung naturgemäß einen hohen Stellenwert einräumt. ${ }^{26}$ Obwohl Sambia eine vergleichsweise durchschnittliche Einkommensverteilung hat, beträgt doch das städtische Einkommen etwa das Dreifache des ländlichen. ${ }^{27}$ Der Dritte Nationale Entwicklungsplan (1979-1983) orientiert sich an den ILO-Empfehlungen zur Grundbedürfnisstrategie und stellt stärker auf die ländliche Entwicklung ab. ${ }^{28}$ Der jüngste ILO-Bericht über Sambia macht vor allem Implementationsschwierigkeiten dafür verantwortlich, daß die ländliche Entwicklung bisher hinter den Planzielen zurückbleibt. ${ }^{29}$

Verstärkte Bemühungen zur ländlichen Entwicklung lassen sich auch als Reaktion auf die ökonomische Situation des Landes ableiten. Der Kupferpreisverfall nach dem Ende des amerikanischen Engagements in Vietnam traf in Sambia einen Industriezweig, der bis dahin $90 \%$ der Deviseneinnahmen des Landes erwirtschaftete. Die darauf folgenden

24 Republic of Zambia, Decentralised Government - Proposals for Integrated Local Government Administration. Lusaka 1978. Republic of Zambia, The District Councillor's Handbook. National Institute for Public Administration, Lusaka 1981, S. 1 f.

25 K. Kaunda, Humanism in Zambia anıl a Guide to it's Implementation. Part I. Lusaka 1968, Part II. Lusaka 1974.

26 International Labour Organisation, Narrowing the Gaps - Planning for Basic Needs and Productive Employment in Zambia. Addis Ababa 1977. Dies., Basic Needs in an Economy under Pressure. Addis Ababa 1981.

27 International Labour Organisation, Basic Needs in an Economy under Pressure, a.a.O., S. 25. Sambia weist für 1974 einen Gini-Koeffizienten von 0.59 auf. Zum Vergleich andere schwarzafrikanische Länder (in Klammern jeweils die Jahreszahl der Angaben): Simbabwe (damals noch Rhodesien): 0.64 (1968), Kenia: 0.61 (1969), Uganda: 0.40 (1970), Sudan: 0.44 (1963), Tansania: 0.53 (1967), Malawi: 0.46 (1969). Vgl. S. Jain, Size Distribution of Income: Compilation of Data. International Bank for Reconstruction and Development. Bank Staff Working Paper No. 190, 1974, zitiert nach H.-R. Hemmer, Wirtschaftsprobleme der Entwicklungsländer, München 1978, S. 26.

28 Republic of Zambia, Third National Development Plan 1979-1983. Office of the President - National Commission for Development Planning, Lusaka 1979.

29 International Labour Organisation, Basic Needs in an Economy under Pressure, a.a.O., S. xiii sowie $107 \mathrm{ff}$. 
Zahlungsbilanzprobleme sollen durch eine höhere eigene Agrarproduktion gemildert werden, die das Land zumindest von Maisimporten (das wichtigste Grundnahrungsmittel der städtischen Arbeiterschaft) unabhängig machen soll.

Die politische Führung hat darüber hinaus mit Loyalitätsverlust zu kämpfen, der sich vor allem in der abnehmenden Mitgliederzahl der einzigen offiziellen Partei Sambias, der United National Independence Party (UNIP), wiederspiegelt. Erklärbar sind diese Verluste unter anderem durch die reale Verschlechterung der Lage auch der städtischen Bevölkerung, die durch ständigen Zustrom vom Land verstärkt wird, was Sambia zum Land mit der Welt höchster Verstädterungsrate macht.

Das Dezentralisierungsprogramm wurde nach mehr als zehnjähriger Debatte am 1. 1. 1981 in Kraft gesetzt. ${ }^{30}$ Die Debatte spielt sich vor dem Hintergrund innenpolitischer Differenzen ab, die hier nur andeutungsweise erörtert werden können.

Die sambische Staatselite besteht im wesentlichen aus zwei Fraktionen, die als "Nationale Politiker" und "Technokraten" bezeichnet werden können. ${ }^{31}$ Die Nationalen Politiker sind meist Angehörige der Unabhängigkeitselite und fühlen sich, oft idealistisch, den egalitären, humanistischen Zielen der Staatsphilosophie verpflichtet. Vielfach ohne formale Berufsausbildung sind sie Verfechter einer Kontrolle von Regierung und Verwaltung durch die Führung der Partei und die treibende Kraft hinter der Einleitung der staatskapitalistischen Phase nach 1968, die mit der Verstaatlichung der wichtigsten Industrien einherging. ${ }^{32}$ Für sie hat nationale, ökonomische Unabhängigkeit einen hohen Stellenwert, die sie auch unter Opfern zu erhalten bereit sind. Sie treten schon früh für ländliche Entwicklung aus moralischer und ethischer Verpflichtung ein. Darüber hinaus fürchten sie die ökonomische und damit politische Abhängigkeit durch Nahrungsmittelimporte und streben daher die Mobilisierung der eigenen agrarischen Ressourcen an. ${ }^{33}$

Die "Technok raten" sind als Fraktion historisch jünger. Sie rekrutieren sich zu großen Teilen aus dem mittleren und oberen Management der Staatsbetriebe, sind private Geschäftsleute oder kommerzielle Farmer. Sie sind meist westlich ausgebildet und orientieren sich dementsprechend an westlich-demokratischen Entwicklungskonzepten. Sie treten für eine profitorientierte Führung der Staatsbetriebe ein und unterstützen Konzepte der internationalen Arbeitsteilung einschließlich ihrer ordnungspolitischen Konsequenzen für die eigene Wirtschaft. Außenpolitische Unabhängigkeit hat für sie einen vergleichsweise geringen Stellenwert. Sie vertreten eine Laissez-faire Politik

30 Republic of Zambia, The Local Administration Act, 1980. Statutory Instrument No. 171 of 1980. Lusaka 1980 .

31 Zur Entwicklung der Klassenstruktur in Sambia. M. Burdette, Where the Copper-Nationalization Worthwhile? University of Zambia/Institute for African Studies, Lusaka 1981, S. 4. I. Scott, Party and Administration under the One-Party State, a.a.O., S. 148 und 158. S. A. Quick, Humanism or Technocracy. Zambias Farming Cooperatives 1965-1972. Lusaka 1978. C. L. Baylies, The State and Class Formation in Zambia. Unveröffentlichte Dissertation. University of Wisconsin 1978. P. Ollawa. Participatory Democracy in Zambia: the Political Economy of National Development, a.a.O., S. $330 \mathrm{fr}$.

32 B. de (jaay Fortman, After Mulungushi. The Economics of Zambian Humanism. Nairobi 1969.

33 R. Hall, The High Price of Principles: Kaunda and the White South. London 1969, S. 6. 
gegenüber dem ländlichen Raum und ziehen es vor, die Ernährung vor allem der städtischen Bevölkerung durch den Aufbau großer, kapitalintensiver Staatsfarmen zu sichern.

Unter den möglichen innenpolitischen Bündnispartnern der beiden Fraktionen sind hier vor allem die Bürokratie, die Gewerkschaften (vor allem die Minenarbeitergewerkschaft) und das Auslandskapital (vor allem im Minensektor) zu nennen. Eine Gruppe von Hinterbänklern im Parlament setzt sich im wesentlichen aus Vertretern der genannten Lobbies zusammen.

Im Verlauf der Debatte um Dezentralisierung hat es wechselnde Koalitionen gegeben. Dennoch sind es die Nationalen Politiker, die das Programm schließlich gegen eine Koalition aus allen anderen Gruppen durchgesetzt haben. ${ }^{34}$

Der Widerstand gegen das Programm erklärt sich teilweise aus seiner inhaltlichen Ausgestaltung, ohne daß jemand ernsthaft den Sinn einer Dezentralisierung öffentlich in Frage stellt. Das Programm sieht vor, die zersplitterten Zuständigkeitsstrukturen, die bis dahin auf die lokale Parteiorganisation, die lokalen Agenten der Ministerialbürokratie und parastaatlichen Unternehmen und die rudimentären Selbstverwaltungskörperschaften verteilt waren, unter Führung eines politischen Distriktgouverneurs zusammenzufassen. Dazu wird das Land einheitlich in 55 (später 57) Distrikte regionalisiert, so daß jeder Distrikt eine Miniaturregierung mit Allzuständigkeit auf seinem Territorium erhält. Das Gesetz sieht vor, daß der Distriktgouverneur vom Präsidenten der Republik unter Zustimmung des Zentralkomitees der Partei eingesetzt wird. Darüber hinaus ist das aktive wie passive Wahlrecht zum Distriktsrat, dem eigentlichen Repräsentativorgan, auf Parteimitglieder beschränkt.

Gegen das Programm in dieser Ausgestaltung opponieren

- die Technokraten, weil sie das Vorhaben für zu kostspielig halten, vor allem was die Personalausstattung der Distrikte angeht;

- die Bürokratie, weil die administrative Tätigkeit der Ministerialbürokratie in den Distrikten der fachlichen und sachlichen Aufsicht einer nach politischen Kriterien eingesetzten Führung unterstellt werden soll;

- die Gewerkschaften, weil mit der Vereinheitlichung der Kommunalverwaltungen der Sonderstatus der Minenstädte aufgehoben wird, der den Minenarbeitern erhebliche materielle Vergünstigungen in Form von Mietsubventionen, geringeren Wasser- und Elektrizitätsgebühren etc. verschafft hatte;

- das Auslandskapital, weil es das mit den Gewerkschaften mühsam ausbalancierte Gleichgewicht zwischen Kapital und organisierter Lohnarbeit durch die Einsetzung eines politischen Führungsbeamten in den Minendistrikten gefährdet sieht; und

- die Hinterbänkler im Parlament, weil sie das oben skizzierte Wahlverfahren als undemokratisch und darüber hinaus als eigentliche Zentralisierung empfinden.

$34 \mathrm{Vgl}$. hierzu und zum folgenden: R. Bodemeyer, Problems of Decentralisation in Zambia. University of Zambia/Institute for African Studies. Lusaka 1981. 


\section{Bewertung und Ausblick}

Mit der Durchsetzung der Dezentralisierung in der vorliegenden Form hat sich die Fraktion der Nationalen Politiker innerhalb der Staatselite gegenüber den Technokraten durchgesetzt. Mit der Dekonzentration der Verwaltung und der gleichzeitigen Zentralisierung und Straffung der politischen Führung verfolgt die siegreiche Fraktion das Ziel einer Stabilisierung der eigenen Machtposition: mit dem Einsetzen der Distriktgouverneure als politische und administrative Führungspersonen schafft die Fraktion die Voraussetzungen für eine Kontrolle der Bürokratie und ihre Instrumentalisierung für die eigenen Interessen.

Dennoch kann von Dezentralisierung angesichts der konkreten Ausgestaltung des Programmes keine Rede sein; es beschränkt sich auf administrative Dekonzentration. Folgt man der These, daß Dekonzentration unter Entwicklungsländerbedingungen nicht hinreicht, Dezentralisierung zu induzieren, ${ }^{35}$ und wird ferner berücksichtigt, daß nur Dezentralisierung aktive Partizipation und damit Grundbedürfnisbefriedigung garantiert, ist eine Verwirklichung der immanenten Ziele des Programmes - bedürfnisorientierte ländliche Entwicklung - ernsthaft gefährdet.

Zwar ist es sicherlich zu früh, die Wirkungen des sambischen Dezentralisierungsprogrammes umfassend zu würdigen. Einige Orientierungspunkte für eine zukünftige Bewertung können jedoch benannt werden.

Die Exekutive steht vor dem Problem, mit dem Dezentralisierungsprogramm zwei Zielsetzungen gleichzeitig verfolgen zu müssen. Das erste Ziel - Mobilisierung der agrarischen Ressourcen - ergibt sich aus dem Programm selbst. Als zweites Ziel kann Legitimationsbeschaffung gelten, das sich aus dem Charakter des Programmes als hohes innenpolitisches Issue ergibt. Dabei müssen auch die hohen politischen Kosten der Verabschiedung des Programmes in seiner jetzigen Form in Rechnung gestellt werden. Beide Ziele sind, wie oben gefolgert wurde, grundsätzlich durch Dezentralisierung zu erreichen. Der Erfolg des Programmes wird dann davon abhängen, wie weit bzw. wie viel faktisch dezentralisiert wird. Ablesbar wird der Grad der Dezentralisierung beispielsweise an dem Umfang öffentlicher Finanzierungskompetenzen, die die zentrale Bürokratie an die Distrikte abtritt, oder auch an der qualitativen und quantitativen Personalausstattung der neuen Distriktregierungen.

Wird zu wenig oder zu wenig weit dezentralisiert, wird der Mobilisierungseffekt weitgehend ausbleiben, d. h. die Loyalitätsbasis der "Nationalen Politiker" und der Staatsklasse als ganzes wird sich nicht verbreitern lassen. In dem Fall wird es sehr schwer sein, das ländliche Klientel der ursprünglich von unten gewachsenen Selbstverwaltungsstrukturen auf die neue, eher politisch legitimierte Führung in den Distrikten zu verpflichten. Die gewünschten Effekte für ländliche und/oder landwirtschaftliche Entwicklung werden dann marginal sein.

$35 \mathrm{Vgl}$. Arbeitsgruppe Stadt-Land-Verflechtung (Hrsg.), Aspekte der Stadt-Land-Beziehungen in Entwicklungsländern. Saarbrücken 1982, S. 105. 
Wird andererseits zu viel, zu weit oder in den "leeren Raum" dezentralisiert, laufen die binnenadministrativen Grundfunktionen Gefahr zusammenzubrechen. Das kann sich in Insubordination der lokalen Ministerienvertreter und der Parastatal-Agenten gegenüber den Distriktgouverneuren äußern. Das Resultat wäre ein Verlust zentralstaatlicher Steuerungsfähigkeit, der nicht durch erhöhte dezentrale Steuerung aufgefangen werden kann. Die wahrscheinliche Folge ist ein erneutes Durchregieren der zentralen Bürokratie, was nicht nur einer Re-Zentralisierung entspräche, sondern der Bürokratie ein vorzügliches Argument an die Hand geben würde, die weitere Implementation des ungeliebten Dezentralisierungsprogrammes aus sachlichen Gründen zu hintertreiben. 


\section{International Interests and Regional Conflicts in Sub-Saharan Africa: The Dynamics of the Decolonization Process in Zimbabwe}

\section{By Gerald Braun}

The majority of conventional studies on international conflict prove to be deficient in at least two respects:

1. they are statically orientated (status quo problem)

2. they concentrate on describing the interests of nation-states (level of analysis problem).

This article attempts to avoid both of these shortcomings. It focusses on the dynamics of conflict in Sub-Saharan Africa by analysing the interests and shifting alliances of the forces involved in the decolonization process in Zimbabwe on a global (USA, USSR, PR China), regional (UK, Frontline States), national (South Africa), and sub-national (Patriotic Front, Smith-Muzorewa Internal Government) level.

The article concludes that some of the fashionable ideas about ostensibly invariable interests and coalitions within the region must be abandoned. For example, the Conservative British Prime Minister Margret Thatcher helped the self-styled Marxist Robert Mugabe to victory in democratic elections; the idealistic human rights policy of the Carter administration achieved its first genuine success since the failure of the Southern Africa policy of the pragmatist Kissinger; the USSR and South Africa who regard themselves as experts on Black African problems completely misjudged the actual balance of power; the economically and socially weak Frontline States proved to be an important power factor in the solution of the conflict.

These are only seeming paradoxes. The increased militarization of the conflict and a growing economic crisis in the region led to its rapid escalation and internationalization. These developments forced the participants to revise their "cost-benefit" political strategies and paved the way for a solution to the conflict by negotiation.

\section{Administration and Basic-Needs oriented Development: The Example Zambia}

\section{By Reinhard Bodemeyer}

The article investigates the impact of basic-needs oriented policy-making on implementation agencies in Third World countries, mostly the public administration. Relations between the administration on one side and state and society on the other side provide the framework for analysis.

The article gives some initial definitions and then focusses on the link between basicneeds oriented policy-making and rural development as a task of agricultural develop- 
ment policies. Active popular participation is described as an indispensable element in basic-needs oriented rural development. Participation in turn requires political and administrative decentralization. Some comments on restrictions on decentralization in developing countries are given in conclusion of the theoretical considerations.

The last section discusses in some detail the strategic value of deconcentrating the administration to improve implementation capacity. Empirical evidence is drawn from the Zambian Decentralization Programme of January 1st, 1981.

\title{
Guerilla Movements in Latin America
}

\author{
By Peter Waldmann
}

The article discusses the conditions of growth and success of guerilla movements in Latin America. It is centred around three topical complexes. First, the principal causes of revolutionary upheaval in Nicaragua under the leadership of the Sandinists are described. In order to test the wider validity of the causal factors delineated with regard to Nicaragua, two further movements are analysed: Venezuela in the early 1960s, and Argentina in the first half of the 1970s. As a result of this comparison, five main conditions are identified for the success, of failure, of guerilla movements in Latin America. The final chapter examines the significance of the Sandinist victory in Nicaragua for the political and military situation in Central America; some conclusions are then offered on the prospects of the conflicts in El Salvador and Guatemala.

\section{Cuba's Legislative Decree No. 50/1982 - Castro's Genuflection Before Capitalism?}

\section{By Ludwig Gramlich}

Cuba enacted a legislative decree on foreign investment in early 1982. Its 50 sections may have been influenced by other joint venture legislation in socialist countries all over the world, but also by genuine Latin American conceptions. The Cuban decree contains rather detailed and relatively liberal provisions for foreign investors which, however, have to be completed by acts of public international law. Particularly in the field of taxation, unilateral fiscal incentives may sooner or later be maintained by tax treaties with home countries of foreign enterprises.

Another significant trait of the new Cuban foreign investment regulations are some provisions on labour law distinguishing between permanent contractual relations of Cuban employers and employees on the one side and temporary work of those employees under direction of foreign entrepreneurs. 